\title{
REVISÃO SISTEMÁTICA SOBRE OS FATORES QUE INFLUENCIAM A CRIATIVIDADE EM AMBIENTES EMPRESARIAIS
}

Lidiana Zocche (lidianazocche) - Universidade Tecnológica Federal do Paraná ,UTFPR, Istefani Carisio de Paula (istefani@producao.ufrgs.br) - Universidade Federal do Rio Grande do Sul, UFRGS,

Vera Lúcia Milani Martins (verinhammartins@gmail.com) - Instituto Federal de Educação, Ciência e Tecnologia do Rio Grande do Sul, IFRS,

Fernando Henrique Lermen (fernando.lermen@unespar.edu.br) - Universidade Estadual do Paraná, UNESPAR.

\section{RESUMO}

Cresce o número de pesquisas que descreve a importância da criatividade para a inovação, mas nem sempre tais estudos exploram as implicações destes achados sobre a prática organizacional. O objetivo deste artigo foi identificar, em pesquisas empíricas realizadas em empresas, evidências sobre os fatores que têm influenciado a criatividade em ambientes empresariais. Para tanto foi realizada uma revisão sistemática da literatura (RSL) visando responder: (i) quais fatores que afetam a criatividade e que foram investigados em seus múltiplos níveis? (ii) quais medidas de criatividade foram mais usadas?. Tendo como base a análise de conteúdo proposto de Myers (2009), foram empregados e os níveis de categorização sugeridos por Kunrath e Paula (2014) e Anderson, Potocnik, Zhou (2014). Utilizou-se na RSL o protocolo PRISMA (Higgins e fGreen, 2011) e 167 artigos provenientes de 4 bases de dados. Os resultados indicaram a predominância dos fatores: estilos de liderança e formação de grupos (nível de grupo); motivação (nível do indivíduo) e clima e estrutura organizacional (nível organizacional). Nas análises multinível, a associação entre as ferramentas de consulta do software NVivo® e Design Structure Matrix (DSM) permitiu verificar que variáveis dos níveis individuo, grupo e organizacional estudadas concomitantemente predominaram. Nas medidas de criatividade houve uma predominância de abordagem "pessoal" e do "ambiente organizacional".

Palavras chave: criatividade, individual, grupo, organizacional, fatores, ambiente industrial 


\section{INTRODUÇÃO}

Sob a perspectiva teórica, a criatividade trata da capacidade de um indivíduo gerar ideias originais e potencialmente úteis, enquanto que a definição de inovação inclui o processo de colocar essas novas ideias em prática (AMABILE, 1988; ZHOU, 1998; ZHOU e SHALLEY, 2003; BIRDI; LEACH; MAGADLEY, 2016). Entretanto, nem sempre o estudo da criatividade esteve voltado para uma visão organizacional. É no contexto dos pesquisadores Woodman, Sawyer, Griffin (1993), Ford (1996) e Drazin, Glynn, Kazanjian (1999) que o presente trabalho se desenvolve: o Modelo Interacionista da Criatividade Organizacional. Esses pesquisadores consolidam o entendimento da importância do contexto na influência da criatividade e apresentam um modelo com múltiplos níveis de análise. Afirmam que a criatividade é produto de uma complexa interação entre pessoas e situações. Ford (1996) já discutia, anos antes, um modelo com múltiplos níveis de análise, mas Drazin, Glynn e Kazanjian (1999) consolidam, a partir da Teoria da Criatividade com Sentido nas Organizações, três níveis denominados individual, de grupo e organizacional.

A literatura apresenta em abundância o estudo dos fatores que afetam a criatividade nas organizações usando tanto modelos teórico/conceituais (Anderson, Potocnik e Zhou, 2014; Zhang, Fan, Zhang, 2015; Xu et al. 2017), quanto investigações empíricas no ambiente das empresas (BIRDI, 2007; GEVERS, 2013; HU, et al. 2017 e PENG, et al. 2014). Algumas dessas pesquisas consideram as análises dos fatores como se fossem independentes, seja por limitações metodológicas ou analíticas. Outros pesquisadores investigam os fatores em seus níveis, buscando identificar quais são as dependências existentes entre eles.

Assim, tomando como premissa que a criatividade é elemento essencial para a inovação nas empresas, os presentes autores se propõem a analisar as pesquisas empíricas realizadas nos ambientes empresariais visando responder três questões de pesquisa: Q1. Quais fatores que afetam a criatividade foram investigados em seus múltiplos níveis? Q2. Quais segmentos industriais foram mais estudados? e Q3. Quais medidas de criatividade foram mais usadas?

A literatura nos mostra que os gestores enfrentam desafios de como criar condições necessárias que possam estender à criatividade dentro das organizações para obterem resultados concretos (CHANG e CHIANG, 2008; MUMFORD et al., 2002). Os autores 
Drazin et al. (1999) comentam que a maioria dos estudos relacionados às questões da criatividade organizacional tendem a explorar formas de ampliar sua manifestação e gestão eficaz. De fato, ainda se busca explicar a manifestação criativa como fruto da interação do indivíduo com fatores externos a ele, visando atender ou gerar novas oportunidades no mercado.

Por isso, é recomendável inicialmente ter o entendimento dos fatores que influenciam a criatividade nas organizações e organizá-las. Os estudos de Kunrah e Paula (2014) e Anderson, Potocnik e Zhou (2014) compilaram informações dispersas na literatura, buscando estruturar os fatores que influenciam a criatividade nos níveis individual, de grupo e organizacional, de forma a construir um conjunto coerente.

Apesar dos estudos citados serem claros, as amostras desta revisão sugeriram que as estruturas organizadas em níveis sejam submetidas a pesquisas complementares, para validação do conjunto dos fatores que influenciam a criatividade nas organizações. O objetivo deste estudo é explorar na literatura acadêmica, especialmente em pesquisas empíricas realizadas em empresas, evidências sobre os fatores que têm significativamente influenciado a criatividade nas organizações.

A contribuição teórica desta RSL é o de fazer um esforço de síntese, apontando os fatores mais estudados e que mais frequentemente têm gerado resultados significativos sobre a criatividade nas empresas, além de explorar as áreas que apresentam o maior número de estudos e apontar os principais modos de mensuração da criatividade nos estudos empíricos. A contribuição prática é o de revelar os segmentos industriais estudados, as implicações dos estudos para a prática destas empresas, criando oportunidade para inferências sobre a gestão da criatividade nas organizações.

\section{METODOLOGIA}

Essa revisão foi conduzida pela adaptação do protocolo PRISMA (Preferred Reporting Items for Systematic Reviews and Meta-Analysis) Moher et al., (2009) e HIGGINS e GREEN (2011). Os artigos científicos sobre a temática foram pesquisados nas bases de dados Web of Science, Science Direct, Scopus e Emerald. Estas bases de dados foram escolhidas por 
cobrirem um número representativo de periódicos de diferentes áreas, com impacto significativo. As buscas foram realizadas no período entre os anos de 2005 a 2017, por concentrarem as pesquisas mais recentes sobre criatividade organizacional.

Partindo das questões de revisão: "quais são as variáveis que influenciam a criatividade nas organizações e como estes fatores estão sendo estudados", as palavras-chave ou termo de busca foram definidos com ajuda do acrônimo PICO, onde: $\mathrm{P}=$ Population ou população envolvida; $\mathrm{I}=$ Intervention ou tipo de intervenção realizada na pesquisa; $\mathrm{C}=$ Control ou controle usado como comparativo ou referência em relação à intervenção; e $\mathrm{O}=$ Outcome ou resultado esperado.

As palavras-chave ou termo de busca e sinônimos foram definidos conforme descrito a seguir: ((industry OR company OR organization OR firm) AND ("Individual creativity" OR "person* creativity" OR "team creativity" OR "group creativity" OR "organization creativity" OR "firm creativity") AND (Elements OR factors OR Expertise OR "creative thinking" OR motivation OR "group training" OR "composition of the group" OR leadership OR "organizational culture" OR "organizational climate” OR "organizational structure" OR “organizational strategy” OR “High Leadership”)).

Foram realizadas 4 etapas durante a RSL. Na primeira fase, que compreende a identificação/seleção, a busca resultou em 1907 artigos distribuídos da seguinte forma: Science Direct (647), Scopus (841), Emerald (347), Web of Science (72), num total de 1907 artigos. Todos eram no idioma inglês dentro do período estabelecido (2005-2017). Esses artigos foram exportados para o software Mendeley para gerenciamento das referências. Foram removidos 40 artigos duplicados. Destes 913 artigos não tratavam de casos empíricos e 787 tratavam da criatividade em ambientes não empresariais, como situações de ensino, acadêmicas. Restaram 167 artigos para a análise de conteúdo. A análise de conteúdo ocorreu por meio da análise construtivista e interpretativa descrita por Bardin (1977); Dhillon (2016); e Myers (2009) desdobada nas etapas de (i) preparação, (ii) categorização, (iii) descrição e (iv) interpretação.

Preparação: Nessa etapa, os artigos foram codificados de acordo com a base de dados de onde provinham, de forma a assegurar a rastreabilidade e inseridos em planilhas Excel@ e no 
Mendeley®. A revisão sistemática foi realizada por dois revisores, os quais usaram como elementos da unitarização, trechos dos artigos que contivessem informações relacionadas com criatividade nos níveis individual, de grupo, organizacional. Foram empregadas como referência para a análise as estruturas de fatores da criatividade adaptada de Kunrath e Paula (2014) e Anderson, Potocnik e Zhou (2014). Como unidades de contexto, foram eleitos: os anos de publicação, os periódicos analisados, áreas organizacionais, os fatores mais estudados e os métodos de mensuração, os quais serviram de referência às unidades de análise, fixando limites contextuais para interpretá-las. Os arquivos em PDF dos 167 artigos foram carregados no software NVivo ${ }^{\circledR}$ assim como em planilha Excel@, contendo a descrição de cada artigo, autor, nome do journal, data, local da pesquisa e tipo de estudo realizado.

Categorização ou classificação das unidades em categorias: nesse caso, os níveis existentes em Anderson, Potocnik e Zhou (2014) e Kunrath e Paula (2014) serviram de referência para a categorização, além das categorias que emergiram da análise textual. Também constituíram nós no NVivo® as dimensões "nível do indivíduo, grupo e organizacional”. Todo o conteúdo dos 167 artigos foi analisado de forma automática no NVivo® e também de forma manual. As consultas realizadas a partir dos dados foram: fatores por países; fatores por metodologia; fatores por elas mesmas; fatores por ano; fatores por abordagem; fatores por dimensão ou nível. A análise manual teve como categorias os termos: nível do individuo; nível de grupo; nível organizacional e outras que emergiram da análise.

Descrição: Para cada uma das categorias foi elaborado um texto síntese em que foi expresso o conjunto de significados presentes nas diversas unidades de análise. Os resultados foram compilados e estão apresentados nos resultados deste artigo. Foi desenvolvida uma consulta no NVivo ${ }^{\circledR}$ onde as categorias encontradas foram cruzadas contra elas mesmos, visando identificar trechos que contemplavam a análise de múltiplos fatores. Os trechos que continham múltiplos fatores (pertencentes a distintos níveis) foram extraídos dos artigos e quantificados. A matriz resultante foi analisada com o Design Structure Matrix (DSM) para formação de agrupamentos.

Interpretação: A interpretação dos achados teve por propósito responder às questões formuladas: (i) quais fatores que afetam a criatividade foram investigados em seus múltiplos 
níveis? (ii) quais medidas de criatividade foram mais usadas. Outros achados bibliométricos foram obtidos e estão descritos nos resultados.

\section{RESULTADOS E DISCUSSÃO}

Visando explorar as publicações sobre o tema criatividade, o procedimento de análise de conteúdo visou encontrar, nos estudos empíricos, evidências sobre os fatores que têm significativamente influenciado a criatividade nas organizações. A distribuição dos 167 artigos incluídos no estudo teve aumento entre os anos de 2010 a 2016, que compreendem 152 estudos do total de 167.

Os países China, Taiwan, EUA e Coreia do Sul, juntos, publicaram praticamente $60 \%$ dos artigos. O crescimento do número de publicações a partir de 2010 pode ser atribuído ao aumento das publicações da China e Taiwan que, juntas, representam 35\%. Os artigos analisados eram provenientes de 38 países diferentes, sendo que 13 desses países compreendem $80 \%$ dos artigos selecionados. Considerando-se que a China é um dos países mais industrializados do mundo, parece coerente um interesse crescente dos pesquisadores pela criatividade no ambiente empresarial e uma ênfase nos segmentos de eletrodomésticos, softwares e eletrônicos. Os artigos estavam distribuídos em 104 diferentes journals (Apêndice A - http://bit.do/eVM3Z), mostrando uma dispersão do tema em áreas de conhecimento também distintas, como tecnologia, inovação, ergonomia, recursos humanos, turismo, engenharia, psicologia, mudança organizacional, inovação entre outras. Houve uma predominância de artigos nos jounals Creativity and innovation Management (10), The Journal of Psychology (6), Journal of Organizational Behavior e Management Decision, com 5 artigos cada um, e apenas um artigo por journal em $70 \%$ dos casos.

\subsection{Fatores que influenciam a criatividade investigadas em seus múltiplos níveis}

Os fatores que influenciam a manifestação criativa dos indivíduos nas organizações foram organizados pelos autores em 54 categorias, agrupadas nos níveis individual, grupo e organizacional, adaptados de Kunrah e Paula (2014) e Anderson, Potocnik, Zhou (2014) (Figura 1). É sugerível não confundir os níveis de categorização dos fatores que influenciam a 
criatividade com os níveis organizacionais da teoria geral da administração (estratégico, tático e operacional), embora eles guardem alguma semelhança. Nos estudos empíricos analisados, foram encontrados fatores presentes nas três dimensões, conforme descrito na Figura 1 (bordas cinza). No entanto, a frequência com que estes fatores apareceram nos trechos de artigo analisados no NVivo® foi distinta e quantificada na figura 1.

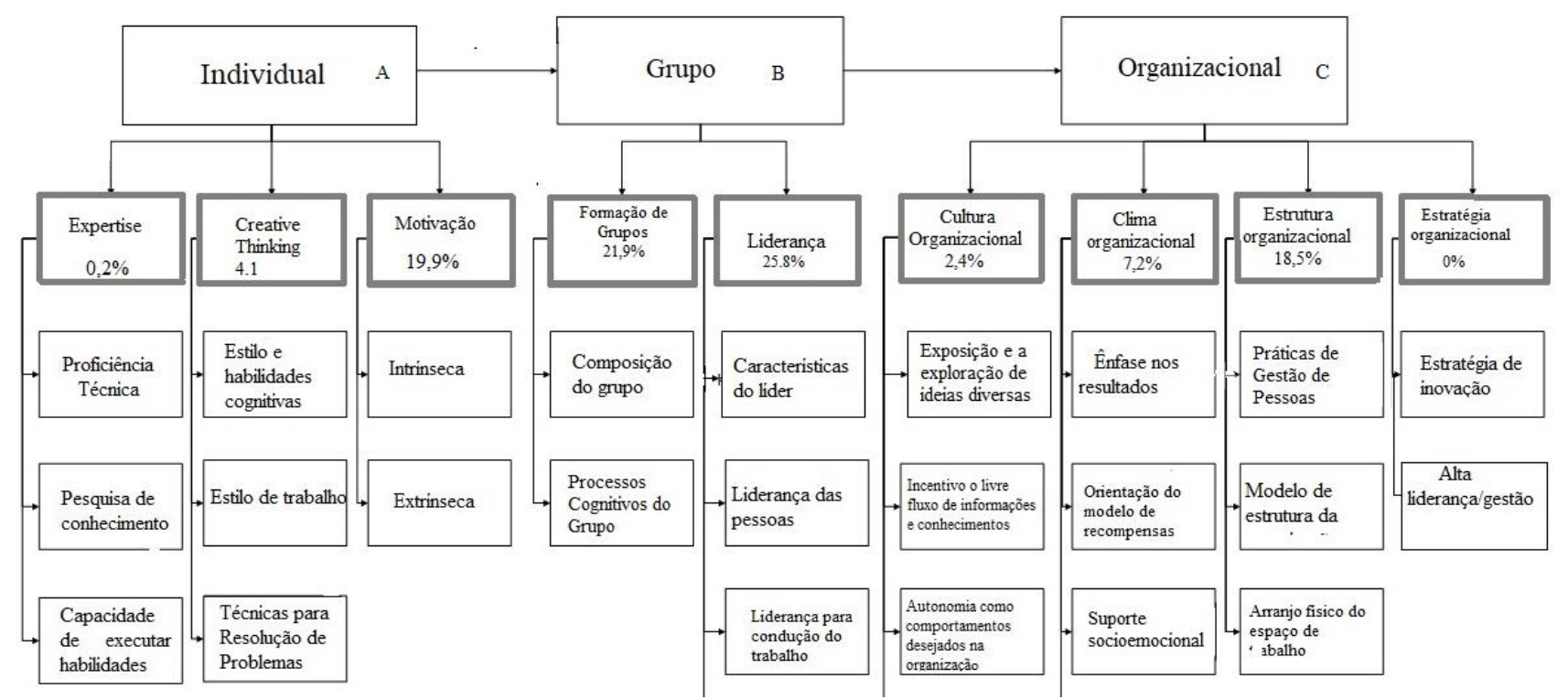

FIGURA 1 - Framework dos níveis e fatores que influenciam a criatividade Fonte: Adaptado de Anderson, Potocnik e Zhou (2014) e Kunrath e Paula (2014)

Embora seja possível quantificar o número de trechos encontrados nos artigos, não interessa realizar uma discussão com base em números absolutos, mas sim pelo indicativo de que alguns fatores foram mais mencionados nos trechos dos artigos analisados do que outros. A variável expertise, por exemplo, estava presente em $0,2 \%$ dos trechos identificados pelo $\mathrm{NVivo}^{\circledR}$, ao passo que liderança compreendeu quase $26 \%$ dos trechos. Essa primeira análise sugere que os fatores de grupo foram mais estudados do que fatores da categoria individual e organizacional. Também sugere uma oportunidade de investigação nessas últimas. Considerando-se que a inovação é fruto do trabalho colaborativo nas empresas, entende-se que estudar a manifestação da criatividade nos grupos se torna relevante, pelo fato de ser considerada uma precursora da inovação.

Já os fatores de formação de grupo e liderança acabam por serem estudados conjuntamente na maioria dos estudos, o que faz sentido pois ambos fazem parte do processo criativo, ou seja, 
os líderes estão focados em melhorar o desempenho e a motivação da formação do grupo. É considerável lembrar que cada nível organizacional apresenta fatores próprios e, à medida que se amplia o olhar nos níveis, observa-se que os fatores ou características do nível anterior são incorporados e interagem com as características e fatores dos níveis subsequentes. Por exemplo, no nível de grupo estão presentes e em plena manifestação as características dos indivíduos que o compõem. A organização por sua vez, influencia grandemente as relações de grupo.

No nível organizacional, os fatores ligados à estratégia tiveram baixa frequência entre os trechos analisados $(0,1 \%)$, embora seja um tema muito relevante. Essa categoria de fatores consiste na análise de que forma as estratégias voltadas para a inovação podem influenciar os resultados da criatividade e, se o envolvimento e valorização da inovação por parte da alta liderança, influenciam os resultados da criatividade e da inovação na empresa. Esses achados sugerem que existe uma oportunidade de pesquisa ainda a ser explorada nesta área, porém maiores investigações devem ser realizadas para tal afirmação, visto que a análise está circunscrita aos artigos desta RSL. No nível organizacional, a variável alta liderança está destacada na figura por não ter sido analisada em nenhum estudo, no entanto Blanchard (2009) ressalta que líderes de alto nível desenvolvem equipes de liderança de elevado desempenho e que contribuem para o maior potencial de cada indivíduo, fator necessário que merece uma atenção maior nas pesquisas.

Horng e Lee (2009) mostram em sua pesquisa a criatividade é alimentada por meio da interação entre indivíduos e seu ambiente, portanto o ambiente é um fator que precisa ser considerado com cuidado para não inibir ou promover a criatividade. Aspectos como (arranjo físico, ergonomia, ambiente de trabalho) se mostram relevantes para promoverem a criatividade dentro da organização (LUKERSMITH e BURGESS-LIMERICK 2013).

Metodologicamente, os pesquisadores tendem a se concentrar em um requisito de criatividade por vez, por ser mais fácil de se lidar, mas ignoram os complexos sistemas socioambientais das organizações que podem influenciar os comportamentos dos funcionários. Essa dimensão dá uma compreensão incompleta em todos os níveis, porque os negócios tipicamente incluem os contextos da empresa, equipe e indivíduo. 
A análise de conteúdo no NVivo® permitiu identificar entre os artigos desta RSL aqueles cujos pesquisadores haviam investigado fatores pertencentes a múltiplos níveis. Uma matriz de categorias x categorias (Figura 2) foi gerada na função "consultas" do software, permitindo quantificar os trechos nos quais os autores estavam analisando mais de uma variável concomitantemente. Em seguida a matriz foi analisada por meio da clusterização típica de uma Design Structure Matrix (DSM).

Um DSM (Figura 2) é uma matriz quadrada em que as células diagonais normalmente representam elementos do sistema (como componentes em um produto, pessoas em uma organização ou atividades em um processo), e as células fora da diagonal representam relações (como dependências, interfaces, interações, etc.) entre os elementos (BROWNING, 2015). Neste estudo, a DSM foi empregada para encontrar os trechos de conteúdos dos artigos da RSL em que haviam sobreposições e interseções em suas codificações. As sobreposições dos trechos codificados indicam estudos multiníveis (Figura 2).

\begin{tabular}{|c|c|c|c|c|c|c|c|c|c|c|}
\hline $\begin{array}{c}\text { DSM } \\
\text { (Variáveis } x \text { Variáveis) }\end{array}$ & 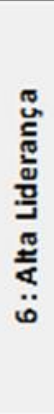 & 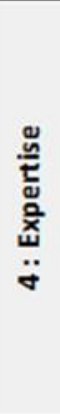 & 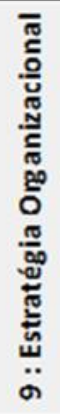 & 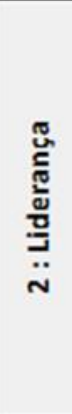 & 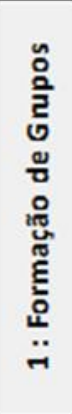 & 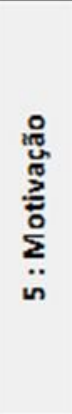 & 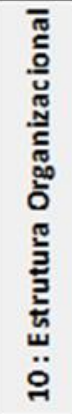 & 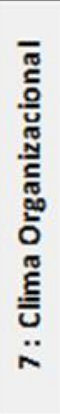 & 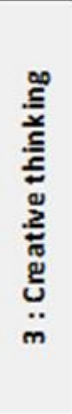 & 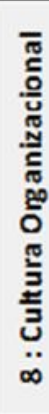 \\
\hline 6 : Alta Liderança & 0 & 0 & 0 & 0 & 0 & 0 & 0 & 0 & 0 & 0 \\
\hline 4: Expertise & 0 & 19 & 0 & 0 & 5 & 3 & 2 & 0 & 4 & 1 \\
\hline 9 : Estratégia Organizacional & 0 & 0 & 10 & 5 & 1 & 1 & 1 & 0 & 0 & 1 \\
\hline 2 : Liderança & 0 & 0 & 5 & 2500 & 502 & 540 & 432 & 206 & 47 & 53 \\
\hline 1 : Formaçăo de Grupos & 0 & 5 & 1 & 502 & 2102 & 312 & 326 & 79 & 65 & 69 \\
\hline 5: Motivação & 0 & 3 & 1 & 540 & 312 & 1908 & 383 & 178 & 88 & 46 \\
\hline 10 : Estrutura Organizacional & 0 & 2 & 1 & 432 & 326 & 383 & 1765 & 187 & 45 & 47 \\
\hline 7 : Clima Organizacional & 0 & 0 & 0 & 206 & 79 & 178 & 187 & 690 & 5 & 28 \\
\hline $3:$ Creative thinking & 0 & 4 & 0 & 47 & 65 & 88 & 45 & 5 & 380 & 3 \\
\hline 8 : Cultura Organizacional & 0 & 1 & 1 & 53 & 6 & 46 & 47 & 28 & 3 & 234 \\
\hline
\end{tabular}

FIGURA 2 - Matriz DSM obtida pela análise dos trechos contendo fatores que influenciam a criatividade nas organizações identificados no software NVivo®

Os valores dentro das células correspondem a sobreposições dos trechos identificados pelo software NVivo® nos 167 artigos analisados. Por exemplo, as células denotam que, nos 
artigos, os trechos contendo as variáveis (2) liderança estão relacionados aos trechos contendo variáveis relacionadas com as variáveis (1) formação de grupos. Também denotam que as variáveis (8) cultura organizacional foram bem menos frequentes do que as variáveis (2) liderança, por exemplo.

Observa-se que 3 blocos de sobreposição de trechos foram denominados A, B e C. O bloco A sugere estudos multinível entre as variáveis liderança, formação de grupos, motivação e estrutura organizacional. O bloco B sugere estudos multiníveis entre as variáveis motivação, estrutura organizacional e clima organizacional e o Bloco $\mathrm{C}$ estudos que relacionam creative thinking e cultura organizacional. As variáveis motivação e estrutura organizacional são os elos de ligação entre os blocos A e B, estando presentes em ambos. Voltando-se aos trechos e artigos da RSL foi possível identificar tais estudos multinível e os achados dos autores, os quais estão resumidos nos Apêndice (http://bit.do/eVM35)..

Os artigos apresentam estudos usando diferentes estilos de liderança, o que dificulta a comparação dos achados, tais como: (i) liderança autêntica ou Leader Member Exchange (LMX) (Xu, et al, 2017; Li et al, 2015 \& Qu, Janssen, Shi (2015); (ii) dual-focused transformational leadership (TFL) (Dong et al, 2017 \& Zhang, Tsui , Wang, 2011; (iii) servant leadership (Jaiswal \& Dhar 2017); (iv) liderança ética (ethical leadership) (Chen \& Hou 2015); (v) liderança empoderadora (empowering leadership) (ZHANG \& BARTOL 2010).

Independentemente do tipo de liderança os estudos mutinível do Bloco A, que envolve as variáveis liderança, motivação, estrutura organizacional e formação de grupos (Figura 2) revelam alguns padrões e implicações práticas que podem ser mencionados:

- (i) Os gestores devem se preocupar em desenvolver lideranças éticas, empoderadoras, humildes que se concentram no bem-estar das pessoas, estimulam a expressão oral, pois elas têm efeito positivo sobre a criatividade do indivíduo. (ii) Os líderes influenciam o compartilhamento de conhecimento nas equipes, que é importante para o desenvolvimento das habilidades e criatividade. (iii) Apesar disso, a troca de informações e criatividade dos funcionários é mediada pela confiança. 
Os artigos relacionados com o Bloco B cujos estudos multinível envolvem as variáveis motivação, estrutura organizacional e clima organizacional (Figura 2), revelam os seguintes achados (mais detalhes sobre achados e autores ver apêndice (http://bit.do/eVM35).

- Existem diferentes culturas organizacionais, por isso os gestores devem identificar tais culturas e auxiliar os colaboradores que se sentem sufocados pela cultura corporativa. Os gestores devem encorajar supervisores, grupos de trabalho, oferecer recursos suficientes e trabalho desafiador, motivando as equipes para o trabalho, de modo a alcançar inovação. Dentre os recursos podem ser incluídos tempo e espaço para mergulhar em seu processo de criação. Os supervisores podem facilitar esse processo personalizando suas práticas de liderança para diferenças individuais no uso do tempo.

- O esforço mencionado depende da motivação intrínseca dos colaboradores. Esta motivação pode se relacionar com o grau de satisfação encontrado no ambiente de trabalho, no atendimento de seus desejos e preferências.

- Embora a orientação para aprender uns com os outros aumente a criatividade organizacional, o intenso uso de TIC reduz a relação entre aquisição de conhecimento e criatividade.

- Além do clima, a cultura organizacional, especialmente fluxo aberto de comunicação, segurança participativa, confiança e respeito pelo indivíduo e abertura ao risco, fortalecem a criatividade.

Dependendo do contexto, a demanda por criatividade se relaciona com o stresse induzido por esta exigência, podendo diminuir o desempenho do serviço. Outro aspecto que pode gerar menor satisfação é uma gestão de RH muito estruturada, embora não necessariamente reduza a criatividade. Lembrando, o Creative thinking ${ }^{l}$ é o componente que pode proporcionar o alto desempenho criativo do indivíduo. É composto do estilo cognitivo, do estilo de trabalho e da capacidade de aplicar técnicas para a exploração de novos caminhos cognitivos. Em relação ao bloco $\mathrm{C}$ os estudos multinível envolvem as variáveis creative thinking e cultura organizacional (Figura 7), e revelam os seguintes achados (http://bit.do/eVM35).

\footnotetext{
${ }^{1}$ Optou-se por manter o termo em seu idioma original porque seu significado extrapola o que seria obtido por sua tradução literal, "pensamento criativo".
} 
- Os gestores devem se ocupar em prover uma qualidade de trabalho e equipe, visto que tem efeitos positivos na relação entre habilidades da equipe e eficiência da equipe.

- Os estudos mostram que capacitação em criatividade em algumas situações facilitam a geração de ideias, o que é mediado pela capacidade criativa e motivação para inovar. No entanto, os treinamentos não eram tão relevantes na fase de implementação dessas ideias. Para a implementação os gestores devem prove ambiente de apoio à gestão e clima adequado.

- Ainda considerando as fases criativas, as habilidades relevantes para a criatividade estão positivamente relacionadas aos índices de geração de ideias, mas não à implementação de ideias. Em vez disso, a experiência profissional dos funcionários, as habilidades operacionais e a motivação para inovar demonstraram um papel mais importante na implementação da ideia.

- Para as atividades de pensamento divergente (exemplo de geração de ideias) uma cultura coletivista gera piores resultados, ao passo que gera melhores resultados em uma cultura individualista.

- O pensamento criativo e a motivação intrínseca mediam completamente a relação entre a liderança transformacional e a criatividade. Verifica-se também que a liderança transformacional tem um efeito relativamente menor no pensamento criativo do que na motivação intrínseca. Além disso, o pensamento criativo tem um efeito maior sobre a criatividade do que a motivação intrínseca.

Analisando-se os conteúdos dos trechos que foram quantificados pelo NVivo® (Figura 2) 35 artigos tratavam de estudos multinível, distribuídos assim: 18 artigos no Bloco A; 11 artigos no Bloco B e 6 artigos no Bloco C. As variáveis motivação e estrutura organizacional estão na interface entre os blocos A e B. A observância da matriz DSM sugere que entre os 167 artigos analisados não houveram análises multinível entre outras variáveis como alta liderança, expertise, estratégia organizacional. Talvez haja uma oportunidade para análises multinível entre estas variáveis em estudos futuros, embora não seja possível fazer uma afirmação categórica sobre isso, pois a análise se circunscreveu aos artigos desta RSL.

\subsection{Quais medidas de criatividade foram mais encontradas nos artigos}


Em relação à segunda questão de pesquisa, Q2, recentemente, Said-Metwaly et al. (2017) realizou uma RSL sobre medidas de criatividade e as classificou em quatro principais abordagens: processo, pessoa, produto e ambiente de trabalho. Apesar de ainda haver vantagens e desvantagens no emprego das medidas citadas por Said-Metwaly et al. (2017), elas expressam um estágio evolutivo da temática mensuração da criatividade, e tal classificação foi adotada na análise dos artigos desta RSL. A Figura 3 demonstra a distribuição dos autores das métricas mais citadas na RSL, tais como relato, análise, contribuição, etc.

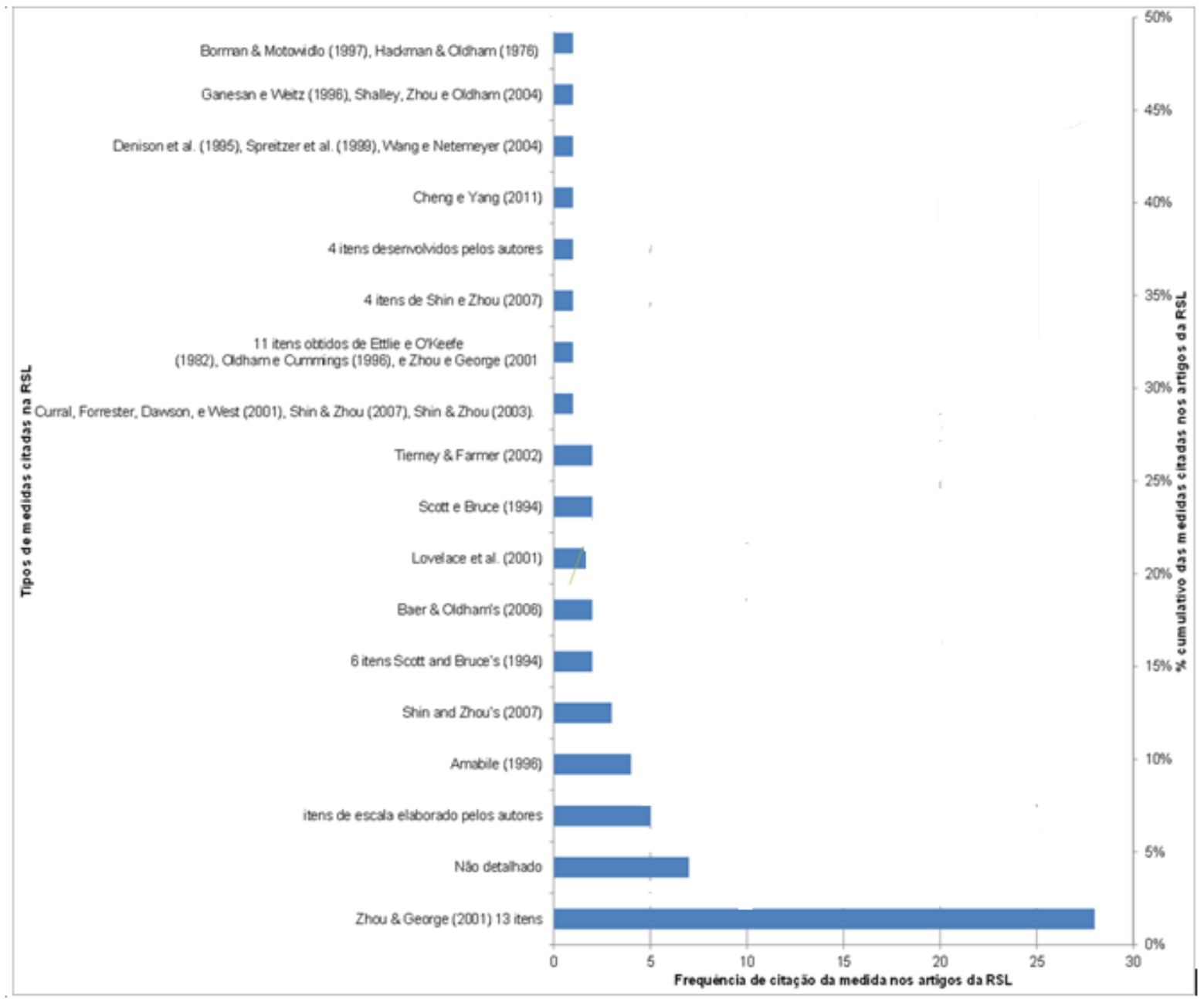

FIGURA 3 - Medidas de criatividade mais citadas nos artigos analisados nesta RSL 
Foram identificadas 98 medidas de criatividade distintas entre os artigos da RSL. Existe uma concentração maior nas mensurações de Zhou e George (2001) (19\%), seguidas por estudos de autores que não detalharam suas métricas e estudos de autores que criaram suas próprias mensurações (8\%). Os estudos/autores que utilizam as escalas de Amabile (1996) (3\%) se mostram presentes, seguidos das medidas de Shin e Zhou (2007) (2\%). As demais medidas citadas nos estudos empíricos, juntas, compreendem $68 \%$.

A escala de 13 itens de Zhou e George (2001) mensura comportamentos que um indivíduo exibe enquanto trabalha para a produção de ideias novas e úteis. A métrica enfatiza a "novidade" das coisas. Essa métrica parece se encaixar melhor na abordagem "pessoas" proposta por Said-- Said-Metwaly et al. (2017). A escala de Amabile (1996) "Assessing the Climate for Creativity" [KEYS], instrumento desenvolvido através de uma colaboração entre Amabile e o Centro de Liderança Criativa e segundo Said-Metwaly et al. (2017), foi classificada como uma abordagem "ambiente de trabalho". Essa escala avalia primariamente a organização e contempla: estímulos, obstáculos e critérios de criatividade e produtividade.

As medidas de Shin e Zhou (2007) parecem estar relacionadas com fatores de controle dos estudos. A criatividade do grupo, por exemplo, pode ser afetada pelo tamanho do grupo (Curral, Forrester, Dawson, e West, 2001), o pertencimento do membro ao grupo ou tipo de grupo (SHIN e ZHOU, 2003). Dessa forma, essas medidas igualmente apareceram com frequência nos estudos empíricos.

Nos estudos de Said-Metwaly et al. (2017), a abordagem por processos foi a mais frequente na sua revisão sistemática de literatura. O propósito inicial desta RSL não era a análise de métricas de criatividade e, nos 167 artigos analisados, diferente dos achados de Said-Metwaly et al. (2017), predominaram métricas da abordagem pessoas (ZHOE e GEORGE, 2001). Apesar da métrica de Zhoe e George (2001) ser uma abordagem mais fácil de ser usada, ela é limitada em seu escopo, por medir a criatividade de forma indireta, ou seja, pela criatividade do indivíduo e não pelos resultados que gera. Além disso, os instrumentos usados geralmente são preenchidos pelo próprio colaborador, numa perspectiva de auto-avaliação. As abordagens que avaliam o ambiente organizacional, como a Amabile (1996) KEYS, por outro lado, também são limitadas em escopo, por serem igualmente medidas indiretas da 
criatividade. Elas permitem avaliar melhorias no ambiente de trabalho para definição de ações corretivas.

\section{CONCLUSÃO}

No que diz respeito a quais fatores afetam a criatividade e quais formas investigadas em seus múltiplos níveis, os estudos empíricos analisados na RSL contiveram fatores dos três níveis (indivíduo, grupo e organizacional) com predominância sobre: os estilos de liderança e formação de grupos (nível de grupo); motivação (nível do indivíduo) e clima e estrutura organizacional (nível organizacional). No que diz respeito às análises multinível, a associação entre as ferramentas de consulta do software NVivo® e DSM mostrou-se um instrumento útil para identificar fatores estudados em múltiplos níveis, o que não é uma tarefa trivial. A tarefa se torna complexa, em virtude da multiplicidade de fatores que influenciam a criatividade, devido à falta de padronização da nomenclatura e dos diferentes contextos de aplicação. Predominaram estudos combinando fatores de liderança e formação de grupos (nível de grupo) x motivação (nível indivíduo) x estrutura organizacional (nível organizacional). Motivação (nível indivíduo) x estrutura organizacional e clima organizacional (nível organizacional) também predominaram. Ainda assim, existe oportunidade para outras combinações de fatores que não foram mencionadas nos estudos empíricos e já comentadas no primeiro parágrafo desse item. Os achados desses estudos multinível geram implicações práticas interessantes para os gestores de empresas e foram descritas na discussão deste artigo.

No que diz respeito às medidas de criatividade mais empregadas nos estudos empíricos, houve uma predominância de abordagem "pessoal" e do "ambiente organizacional". De certa forma, parece adequado, uma vez que os estudos eram empíricos. No entanto, essas medidas são indiretas, não avaliam o impacto da criatividade diretamente, nem seu efeito sobre os resultados da organização. A criatividade é uma qualidade multifacetada. Especialmente quando se deseja medir a criatividade em termos de resultado para organização faz sentido associar métodos de medição, havendo, portanto, uma oportunidade para se explorar melhor essa questão. 
Por fim, houve uma surpresa quanto à diversidade de áreas onde os estudos estão sendo realizados (hospitais, alimentícia, têxtil, energia, militar, química, TI, manufatura, turismo, hotelaria, etc.); nas áreas criativas como design e publicidade, houveram poucos estudos, sendo a área de TI uma das áreas predominantes.

Entende-se que a contribuição deste artigo para a área de conhecimento foi explorar o subconjunto de estudos empíricos na área da criatividade revelando sua abrangência e tendências. Sendo a criatividade precursora da inovação e sendo a inovação tão vital para a sobrevivência das organizações, faz sentido que os pesquisadores se debrucem mais sobre o ambiente organizacional, buscando compreender de que forma a criatividade ali se manifesta. Muito trabalho ainda precisa ser realizado, desde a definição clara da criatividade até a sua mensuração. Fatores ainda podem ser explorados em seus múltiplos níveis e seus impactos avaliados. Também diferentes segmentos industriais merecem atenção, sob a premissa de que a criatividade é uma manifestação natural e que pode influenciar qualquer ambiente organizacional.

\section{REFERENCIAS}

AMABILE, T. M. A model of creativity and innovation in organizations. Research in Organizational Behavior, v.10, p.123-167, 1988.

AMABILE, T.M. Creativity and Innovation in Organizations. Harvard Business School Background Note 396-239, 1996

ANDERSON, N.; POTOCNIK, K.; ZHOU, J. Innovation and Creativity in Organizations: A State-of-theScience Review, Prospective Commentary, and Guiding Framework. Journal of Management, v. 40 (5), 2014.

BLANCHARD, K. Leading at a Higher Level, Revised and Expanded Edition: Blanchard on Leadership and Creating High Performing Organizations. 385f. Pearson Prentice Hall; Edição: 2, 2009.

BIRDI, KAMAL. A Lighthouse in the Desert? Evaluating the Effects of Creativit Training on Employee Innovation. Journal of Creative Behavior, v. 41 (4), 2017.

BIRDI, K.; LEACH, D.; MAGADLEY, M.The Relationship of Individual Capabilities and Environmental Support with Different Facets of Designers' Innovative Behavior. The Journal Product Innovation Management, v. 33 (1), 2016.

BIRDI, KAMAL. A Lighthouse In The Desert? Evaluating The Effects Of Creativity Training On Employee Innovation. Journal Of Creative Behavior, V. 41 (4), 2007.

BROWNING, T. (2015). Design Structure Matrix Extensions and Innovations: A Survey and New Opportunities. IEEE Transactions on Engineering Management, v. 63(1), p.1-26, 2015. 
CHANG, WEN-CHIH; CHIANG, ZUN-HWA. A study on how to elevate organisational creativity in taiwanese design organization. International Journal of Innovation Management, v. 12, n. 4, 2008.

CURRAL, L. A. FORRESTER, R.H. DAWSON, J. F. WEST, M. It's what you do and the way that you do it: Team task, team size, and innovation-related group processes. European Journal of Work and Organizational Psychology, 2001.

FORD, C. M. A theory of individual creativity in multiple social domains. Academy of Management Review, v. 21, p. 1112-1134. 1996.

GEVERS, J. M. P.; DEMEROUTI, E. How supervisors' reminders relate to subordinates' absorption and creativity. Journal of Managerial Psychology, v. 28 (6), p. 677 - 698, 2013.

Higgins, J. P.T. e Green, S. Cochrane Handbook for Systematic Reviews of Interventions. Version 5.1.0. The Cochrane Collaboration. 2011.

HORNG, J. S.; LEE, Y. C. What environmental factors influence creative culinary studies?. International Journal of Contemporary Hospitality Management, v. 21, n.1, 2009.

HU, N.; CHEN, GU, J. HUANG, S.; LIU, H. Conflict and creativity in inter-organizational teams the modwrating role of shared leadership. Journal of Management, v.28 (1), 2017.

KUNRATH, S. E.; PAULA, I. C. Identificação e Organização de Fatores que Influenciam a Criatividade nas Organizações. In: $4^{\circ}$ Congresso Internacional de Conhecimento e Inovação, 2014, Loja. $4^{\circ}$ Congresso Internacional de Conhecimento e Inovação, 2014.

LUKERSMITH, S; BURGESS-LIMERICK, R. The perceived importance and the presence of creative potential in the health professional's work environment. Ergonomics, v.56 (6), 2013.

MOHER, D.; LIBERATI, A.; TETZLAFF, J.; ALTMAN D. G. The PRISMA Group. Preferred reporting items for systematic reviews and meta-analyses: The PRISMA Statement", PLoS Med, v.6 (7), 2009.

PENG, J.; ZHANG, G.; FU, Z.; TAN, Y. (2014). An empirical investigation on organizational innovation and individual creativity. Information Systems and e-Business Management, v. 12 (3).

SAID- METWALY, S.; KYNDT, E. E NOORTGATE, W. V. D. (2017). Approaches to Measuring Creativity: A Systematic Literature Review. Creativity Theories Research Applications, 4 (2), 2017.

SHIN, S. J., \& ZHOU, J. When Is Educational Specialization Heterogeneity Related to Creativity in Research and Development Teams? Transformational Leadership as a Moderator. Journal of Applied Psychology,v. 92, p.1709-1721, 2007.

WOODMAN, R. W.; SAWYER, J. E.; GRIFFIN, R. W. Toward a Theory of Organizational Creativity. Academy of Management Review, v. 18, n. 2, p. 293-321, 1993.

XU, B. D; ZHAO, S. K.; LI, C. R.; LIN, C. J. Authentic leadership and employee creativity: testing the multilevel mediation model. Leadership e Organization Development Journal, v. 38 (3), 2017.

ZHANG, J.; FAN, ZHANG. The role of power motivation in creativity: A moderated mediation model. Social Behavior and Personality, 43 (4), 2015.

ZHOU, J., SHALLEY, C. E. Research on employee creativity: a critical review and directions for future research. Research in Personnel and Human Resource Management, v. 22, p. 165-217, 2003. 
11 a 13 de setembro de 2019 - Universidade de Brasília UnB

ZHOU, J. AND GEORGE, J.M. When Job Dissatisfaction Leads to Creativity: Encouraging the Expression of Voice. Academy of Management Journal, v.44, p.682-69, 2001. 